\title{
Review of Developments in Methods of Reconfiguration of Distribution Networks
}

\author{
Prof. A. B. Kansae ${ }^{1}$, Dr. S. R. Gengaje ${ }^{2}$ \\ ${ }^{1}$ Walchand Institute of Technology, Solapur, Maharashtra, India
}

\begin{abstract}
Distribution networks are configured radially. Their configurations may be varied with manual or automatic switching operations so that all of the loads are supplied and reduce power loss, increase system security, and enhance power quality. Reconfiguration also relieves the overloading of the network components. The change in network configuration is performed by opening sectionalizing (normally closed) and closing tie (normally open) switches of the network. These switchings are performed in such a way that the radiality of the network is maintained and all of the loads are energized. Obviously, the greater the number of switches is, the greater the possibilities are for reconfiguration and the better the effects are. This paper presents a review of developments and improvements in distribution network reconfiguration methods which includes multi-agent and genetic algorithm approach.
\end{abstract}

Keywords: Distribution networks, reconfiguration, genetic algorithm, multiagent.

\section{Introduction}

Distribution systems consist of groups of interconnected radial circuits. The configuration may be varied via switching operations to transfer loads among the feeders. Two types of switches are used in primary distribution systems. They are normally closed switches (sectionalizing switches) or normally open switches (tie switches). Both types are designed for both protection and configuration management. Distribution network reconfiguration is a process that consists of changing the status of the network switches in order to resupply the non-energized areas after a fault occurrence, or optimize given criteria. The reconfiguration problem of radial power distribution networks may be stated as follows: given a load profile for a distribution network with a number of tie lines and switching points, find a radial configuration for the network, which minimizes the network losses, supply the critical loads at acceptable voltage levels.

In recent years, considerable research has been conducted for loss minimization in the area of network reconfiguration of distribution systems. Distribution system reconfiguration for loss reduction was first proposed by Merlin and Back. They have used a branch-and-bound-type optimization technique to determine the minimum loss configuration.

The aim of this paper is to present review of developments and improvements in distribution network reconfiguration methods which includes multi-agent and genetic algorithm approach.

\section{Multi-Agent Technology}

Li et al. [1] proposed a novel systematic algorithm for the dynamic reconfiguration of the distribution network based on multi-agent system. In which, one day was divided into several time intervals and each was managed by a work agent. An improved hybrid particle swarm optimization algorithm was applied in each time interval to produce a solution set which included some enough good solutions. Then the coordination agent would select a solution from each work agent's solution set, and coordinate them until the number constraint of switching operations was satisfied. The whole architecture of the multi-agent system was presented, and the function modules of the work agent and coordination agent were also discussed.

Proposed novel systematic algorithm for dynamic reconfiguration of the distribution network minimizes line losses. The dynamic reconfiguration was decomposed into a series of static ones, and a series of solution sets were calculated. Then a solution was chosen in each set and all the chosen solutions belong to different solution sets composed the dynamic reconfiguration result. The approach was easily realized via MAS, and the computer simulations showed the effectiveness. In the algorithm if the maximum allowed number of switching operations were changed, only the coordination need to be carried out again. And in the coordination process, along with the reduction of the allowed number of switching operations, the loss increase quantity and the time interval that abandoned the optimal solution could be both obtained. If the loss increased remarkably the allowed number of switching operations should be changed larger than the current value. Conversely, if the loss increased only a little, it should be reduced.

The distribution network re-configuration is a process that consists of changing the status of the network switches for rerouting the power after a fault occurrence, or to optimize some given criteria. Traditionally, feeder reconfiguration is a complex combinatorial and constrained optimization problem because of the numerous combinations of candidate switches. Chauhan et al. [2] proposed a multi-agent based system for distribution network reconfiguration. The system consists of load agents and switch agents located at the loads and switches in the network and a global agent located at the substation. The objective was to supply the critical loads while maintaining an acceptable system voltage profile. Simulation results presented for a typical distribution system named Circuit of the Future (CoF), developed by Southern California Edison (SCE). 


\section{International Journal of Science and Research (IJSR) \\ ISSN (Online): 2319-7064}

Index Copernicus Value (2013): 6.14 | Impact Factor (2014): 5.611

The approach deploys multi-agent system in conjunction with graph theory algorithms to reconfigure the system. The proposed Multi-agent system makes use of both centralized and decentralized methods to make up for the disadvantages of each method. The proposed model of MAS is tested on proto-type distribution network named Circuit of the Future $(\mathrm{CoF})$. The team is developing multi-agent deployments and application algorithms to reconfigure the system to supply the critical loads and to maintain the good voltage profile across the network even in the event of faults. The fault reconfiguration algorithm makes use of shunt compensation and priority based load shedding in order to control the voltage. The fault reconfiguration algorithm proposed in this paper chooses the best possible switching configuration in regards to voltage profile and real power loss in the system. The results obtained are very promising and show superior ability of Multi-agent systems in the field of fault detection and reconfiguration. The next stage of the work will be focused on reconfiguration in a even more complicated smart system and finding better interface between different simulation platforms for efficiency improvement.

Lin et al. [3] proposed a novel method is proposed to solve the distribution network reconfiguration. The proposed method combines the binary particle swarm optimization (BPSO) with discrete particle swarm optimization (DPSO) and multi-agent system (MAS). It also incorporates selflearning mechanism and the survival of the fittest which is a part of natural selection for reconfigure distribution network. In the algorithm, each particle is thought to be an agent and all agents live in a lattice-like environment. The competition and cooperation operator is preformed on every agent. If the agent is a loser, a new agent will occupy its lattice-point which is determined by BPSO and DPSO. If the agent is a winner, it can perform the self-learning mechanism, so as to obtain the better fitness value. Finally the next iteration particles are determined by the fitness value of previous particles, according to the survival of the fittest mechanism. The result showed that the method has superior features, including good computation efficiency, good convergence characteristics, and high-quality solutions etc.

In MAS, to build a lattice-like environment, all agents improve their communication by the competitive and cooperative operation, unlike individual agents in PSO. In this process, it is in favor of converged to the global optimized results. Those particles will divide into two parts through the competition and cooperation operator. The losers will update by HPSO, which can avoid premature convergence and to get the best solution in a global search. The winners will update by self-learning mechanism.

\section{Genetic Algorithm}

Paar et al. [4] presented one possible solution to find optimal configuration of switches in the meshed distribution network which leads to minimal interruption payment for selected continuity standard - Finnish, Portuguese and modified Portuguese. For its suitable properties, simple genetic algorithm was chosen as the optimization. Functionality of this approach is demonstrated on the example of a real cable MV distribution network.

The Figure 1 shows the trends of penalizations and SAIDI values during optimization. Average values of SAIDI for Portuguese (PT) and Modified Portuguese (X) are very similar, on the contrary to Finish (FI) penalization model. Finish model also has the highest value of penalization and modified Portuguese achieves low order values compare to others standards. Considering the results, graphs reveal that SAIFI and SAIDI values do not have to correspond directly to the values of penalizations. The solution with minimal penalization does not determine that SAIDI and SAIFI are minimal as well. Moreover the results showed that different penalization models lead to various optimal outputs. These conclusions are in accordance with results.

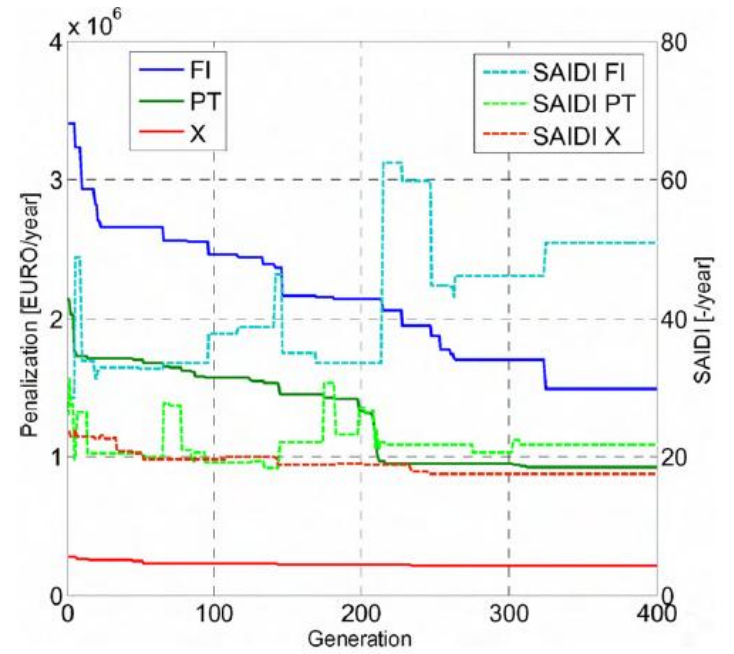

Figure 1: Comparison of Penalization and SAIDI among all standards

Sawa et al. [5] presented a new Particle Swarm Optimization (PSO) for optimal radial network reconfiguration. Network reconfiguration is a combinatorial problem. The combination of search space increases exponentially with the number of switches. Fixed loop coding structure is introduced to reduce the number of combinations. The feature of this method is to introduce mutation operation for discrete decimal problem to PSO. This method, discrete decimal PSO with mutation (DDM-PSO), is applied to a test system with 37 nodes and 63 branches. The proposed method improves the maximum arrival number at the optimal solution from 8 to 17 compared to PSO without mutation in twenty trials. This number of DDM-PSO is also more than that of GA, 10.

An innovative radial network reconfiguration method has been developed using the discrete decimal mutant PSO (DDM-PSO). The feature of this method is to introduce mutation operation and a fixed loop coding structure. This method was applied to a test system with 37 nodes and 63 branches. The proposed method improves the maximum arrival number at the optimal solution from 8 to 17 compared to PSO without mutation. This showed the effectiveness of the developed method.

Reconfiguration of radial distribution system is the significant way of altering the flow of power through lines.

\section{Volume 5 Issue 1 January 2016}




\section{International Journal of Science and Research (IJSR) \\ ISSN (Online): 2319-7064}

Index Copernicus Value (2013): 6.14 | Impact Factor (2014): 5.611

This altered flow changes the real power losses, reactive power losses and voltage profiles. Privatized RDS need to operate profitably with minimum operational losses and power quality. Envisaging such a prospect, Venkatesh et al. [6] focused on the aspects of loss minimization and voltage enhancement of RDS by artificial intelligence methods. A sample 33-bus system and 69-bus system are chosen for the study and the results compared.

This method used two algorithms namely FMGA and EP to reconfigure the RDS by minimizing the real and reactive power losses and at the same time improving the power quality. The comparison of results with both the algorithms showed that FMGA is able to find potentially good switching options. EP has good solution but it takes a longer time to converge. In FMGA, more potential solutions that are found quickly and crossover exploits these new candidate solutions to find the best solution quickly.

The topology of an Electrical Distribution System (EDS) can be suitably modified to minimize the real losses. Basically, losses in an EDS arise due to two factors: 1) fault in the network 2) overload in the feeders. EDS is normally unevenly loaded and hence often need load balancing, which can also be done by reconfiguring the network like changing the status of both sectionalizing and Tie switches. They presented a new approach for optimal reconfiguration of a radial Electrical Distribution Network based on the advanced genetic algorithm using improved methods of selection, crossover and fitness function to determine the optimal configuration path. The opened switches are taken into consideration for crossover process. After obtaining number of solutions (off springs) from the combinational analysis, the optimal solution is selected based on the fitness function, i.e., the solution having the minimal power losses.

Ravibabu et al. [7] presented an advanced method to study the electrical distribution network through feeder reconfiguration using Improved Genetic Algorithm (IGA). The Distribution Network Feeder Reconfiguration (DNRC) is modified efficiently using some improvements in crossover and mutation. From several case studies and comparison with other methods including heuristic approach, it is proved that Improved Genetic Algorithm is more efficient and satisfies the conditions of the global optima.

\section{Conclusion}

This paper presents review of developments in reconfiguration methods of distribution networks. The results presented showed that the multiagent systems have superior ability in the field of fault detection and reconfiguration. A study on electrical distribution using improved Genetic Algorithm is proved to be efficient and satisfies the conditions of the global optima.

\section{References}

[1] Zhenkun Li, Xingying Chen, Kun Yu, Bo Zhao, and Haoming Liu, "Novel Approach for Dynamic Reconfiguration of the Distribution Network via Multi-
Agent System”, IEEE Xplore, page no. 1305-1311, 2008.

[2] Sridhar Chouhan, Hui. Wan, H.J.Lai, Ali Feliachi, M. A. Choudhry, "Intelligent Reconfiguration of Smart Distribution Network using Multi-Agent Technology", IEEE Xplore, 2009.

[3] Hongbin Sun, Yongsheng Ding, "Network Reconfiguration of Distribution System Using Fuzzy Preferences Multi- Objective Approach", 2nd International Asia Conference on Informatics in Control, Automation and Robotics, page no. 93-96, 2010.

[4] Martin PAAR, Pekka VERHO, Petr TOMAN, "An Application Of Genetic Algorithms To The Distribution Network Reconfiguration For Minimization Of Interruption Costs", 20th International Conference on Electricity Distribution, 2009.

[5] T. Sawa, "Radial Network Reconfiguration Method in Distribution System using Mutation Particle Swarm Optimization", IEEE Bucharest Power Tech Conference, page no. 1-6, 2009.

[6] B. Venkatesh, S. Chandramohan, N. Kayalvizhi, R.P. Kumudini Devi, "Optimal Reconfiguration of Radial Distribution System Using Artificial Intelligence Methods" page no. 660-665, 2009.

[7] P. Ravibabu, M.V.S.Ramya, R.Sandeep, M.V.Karthik, S.Harsha, "Implementation of Improved Genetic Algorithm in Distribution System with Feeder Reconfiguration to Minimize Real Power Losses", 2nd International Conference on Computer Engineering and Technology, Vol. 4, page no. 320-323,2010.

\section{Author Profile}

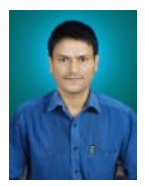

Prof. A. B. Kanase received his M.E in Electronics Engineering from Shivaji University, Kolhapur, Maharashtra State, India. He is currently working as Principal in Karmayogi Polytechnic College, Pandharpur, Dist. Solapur, Maharashtra State, India. His research interest includes Power Electronics, Optimization Techniques and Automation.

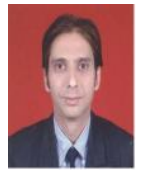

Dr. S. R. Gengaje received his $\mathrm{PhD}$ in Electronics Engineering from Shivaji University, Kolhapur, Maharashtra State, India. He is currently working as Professor and Head, Department of Electronics in Walchand Institute of Technology, Solapur, Maharashtra State, India. His research interest includes Soft Computing, Image and Video Processing and Telecommunication. 